Revue internationale P.M.E.

Économie et gestion de la petite et moyenne entreprise

\title{
Capitalisation des entreprises québécoises: évolution et état de la situation
}

\section{Jean Marc Suret et Laurence Arnoux}

Volume 8, numéro 3-4, 1995

URI : https://id.erudit.org/iderudit/1008363ar

DOI : https://doi.org/10.7202/1008363ar

Aller au sommaire du numéro

Éditeur(s)

Presses de l’Université du Québec

ISSN

0776-5436 (imprimé)

1918-9699 (numérique)

Découvrir la revue

Citer cet article

Suret, J. \& Arnoux, L. (1995). Capitalisation des entreprises québécoises: évolution et état de la situation. Revue internationale P.M.E., 8(3-4), 165-179. https://doi.org/10.7202/1008363ar
Résumé de l'article

À l'aide de données tirées des statistiques fiscales du gouvernement du Québec, nous montrons ici qu'il n'existe pas de relation systématique entre la taille et rendettement des entreprises québécoises. Les entreprises les moins endettées sont celles dont l'actif total se situe entre 100000 et 1 million de dollars ainsi que les grandes entreprises, dont l'actif dépasse 50 millions de dollars. Inversement, les entreprises dont l'actif se situe entre 1 et 50 millions de dollars sont les plus fortement endettées et le phénomène semble particulièrement évident pour les entreprises de 5 à 15 millions de dollars d'actif. L'endettement élevé serait donc davantage une caractéristique des entreprises de taille moyenne que des plus petites. Par ailleurs, il existe une relation très nette entre la capacité à générer des bénéfices et l'endettement. À taille égale, la différence entre les niveaux d'endettement des sociétés imposables et non imposables atteint 25 points de pourcentage, alors que les différences dues à la taille ne dépassent pas 7 points de pourcentage.
Ce document est protégé par la loi sur le droit d'auteur. L’utilisation des services d’Érudit (y compris la reproduction) est assujettie à sa politique d'utilisation que vous pouvez consulter en ligne.

https://apropos.erudit.org/fr/usagers/politique-dutilisation/ 


\title{
Capitalisation des entreprises québécoises: évolution et état de la situation
}

Jean Marc SURET*

Laurence ARNOUX**

CIRANO, Montréal

MOTS CLÉS

PME - Financement - Endettement

\begin{abstract}
RÉSUMÉ
À l'aide de données tirées des statistiques fiscales du gouvernement du Québec, nous montrons ici qu'il n'existe pas de relation systématique entre la taille et l'endettement des entreprises québécoises. Les entreprises les moins endettées sont celles dont l'actif total se situe entre 100000 et 1 million de dollars ainsi que les grandes entreprises, dont l'actif dépasse 50 millions de dollars. Inversement, les entreprises dont l'actif se situe entre 1 et 50 millions de dollars sont les plus fortement endettées et le phénomène semble particulièrement évident pour les entreprises de 5 à 15 millions de dollars d'actif. L'endettement élevé serait donc davantage une caractéristique des entreprises de taille moyenne que des plus petites. Par ailleurs, il existe une relation très nette entre la capacité à générer des bénéfices et l'endettement. À taille égale, la différence entre les niveaux d'endettement des sociétés imposables et non imposables atteint 25 points de pourcentage, alors que les différences dues à la taille ne dépassent pas 7 points de pourcentage.
\end{abstract}

* Jean Marc Suret détient un doctorat en administration des affaires de l'Université Laval. Il est professeur titulaire au Département de finance de cette université et directeur de recherche au Centre interuniversitaire de recherche en analyse des organisations (CIRANO) de Montréal. Il s'intéresse à la problématique du financement des entreprises, à l'analyse financière et à l'efficience des marchés. Ses articles ont été publiés notamment dans Contemporary Accounting Research. The Financial Review, The Journal of Financial Research, The Geneva Papers on Risk an Insurance, The Canadian Journal of Administrative Sciences, Finance et l'Actualité Économique. Adresse : Centre interuniversitaire de recherche en analyse des organisations, 2020, rue University, Montréal (Québec) H3A 2A5.

** Laurence Arnoux détient un MBA en administration des affaires de l'Université Laval, était stagiaire de recherche au CIRANO. Adresse: Centre interuniversitaire de recherche en analyse des organisations, 2020, rue University, Montréal (Québec) H3A 2 A5. 


\section{ABSTRACT}

This study, prepared using tax data from the Province of Québec, demonstrates that there is no systematic relationship between company size and leverage. In Québec, the smallest (defined as having between $\$ 100000$ and $\$ 1 \mathrm{M}$ in assets) and the largest (having more than \$50M in assets) companies are the least levered. Inversely, companies having between $\$ 1 \mathrm{M}$ and $\$ 50 \mathrm{M}$ in assets have the greatest leverage, with those companies having between $\$ 5 M$ and $\$ 15 \mathrm{M}$ in assets being the most heavily in debt. Consequently, high debt ratios appear to be a characteristic of medium-sized rather than small companies. In addition, we find a clear positive relationship between leverage and propensity to pay taxes. For companies in the same size category, the difference in the proportion of assets financed with debt between tax-paying and non tax-paying companies is 25 percentage points; the size categories themselves only explain a maximum of 7 percentage points of the proportion of debt financing used.

\section{RESUMEN}

A partir de la información obtenida de las estadísticas fiscales del gobierno de Quebec, en el presente estudio se demuestra que no existe ninguna relación sistemática entre el tamaño y el nivel de endeudamiento de las empresas de Quebec. Las empresas menos endeudadas son las pequeñas empresas, aquéllas cuyo activo total se sitúa entre los 100,000 y el millón de dólares, y las grandes empresas, aquéllas cuyo activo total se sitúa a un nivel superior de 50 millones de dólares. Por el contrario, las empresas cuyo activo se encuentra entre uno y 50 millones de dólares son las más endeudadas; fenómeno bastante marcado para aquéllas que poseen un activo de cinco a 15 millones de dólares. El nivel de endeudamiento elevado será, más que todo, una característica de las empresas de tamaño medio que de las pequeñas empresas. Por otro lado, existe una relación marcada entre la rentabilidad y el endeudamiento. Considerando a empresas de la misma categoría, la diferencia entre los niveles de endeudamiento de empresas sujetas a impuesto y las no sujetas a impuesto llega a ser de 25 puntos porcentuales; mientras que la diferencia debida al tamaño no es mayor que siete puntos porcentuales.

\section{Introduction}

Au Québec, comme dans l'ensemble du Canada, le niveau d'endettement des PME est à l'origine de nombreux programmes gouvernementaux. Suivant le discours généralement admis, les PME seraient sous-capitalisées. Par exemple, le discours sur le Budget 1983-1984 rapporte que «le problème de la souscapitalisation des entreprises reste endémique. Il faut, de toute évidence, inciter davantage, d'une part, les entreprises à augmenter leur capital-actions, et d'autre part, les particuliers à acheter ces actions» (p. 22). Même si les travaux récents mentionnent un problème de financement plus localisé, touchant surtout les entreprises des secteurs technologiques (Conseil de la science et de la technologie, 1993), les actions et propositions d'action des gouvernements 
en ce domaine visent encore souvent à augmenter l'offre de capital de risque afin de permettre aux PME de se capitaliser plus facilement.

La survivance de cette notion de sous-capitalisation des PME est surprenante, car il ne semble pas exister de travaux démontrant que les entreprises de petite taille sont effectivement plus endettées que les grandes entreprises. La plupart des écrits se réfèrent à quelques tableaux tirés des travaux de Gagnon et Papillon (1984), élaborés à partir de données datant de 1977. Ces auteurs ne concluaient d'ailleurs pas que les PME étaient surendettées. Cette apparente contradiction, la nécessité de mettre à jour les données et l'importance des enjeux économiques qu'implique le financement des entreprises justifient cette étude. Elle cherche à déterminer dans quelle mesure la souscapitalisation ${ }^{1}$ existe et analyse également l'évolution de l'endettement des entreprises au cours d'une période qui a vu la création de très nombreux programmes gouvernementaux destinés à favoriser la capitalisation des entreprises de petite taille.

L'étude des caractéristiques financières des petites entreprises ne peut se faire à partir des bases de données conventionnelles, généralement restreintes aux sociétés inscrites en Bourse et soumises aux exigences de divulgation. Il faut donc utiliser des données agrégées, rassemblées par les organismes officiels. L'étude présentée ici repose sur celles de Revenu Québec ${ }^{2}$. L'utilisation de ce type d'informations présente des avantages, mais limite également la portée des résultats. La description des données et des limites qu'elles imposent fait l'objet de la première section. La seconde partie est consacrée à l'étude de la situation de l'endettement en 1990, dernière année pour laquelle les données sont disponibles. La troisième partie présente l'évolution de l'endettement de 1979 à 1990.

\section{Les données}

L'étude est basée sur les statistiques financières et fiscales du ministère du Revenu du Québec et porte uniquement sur les sociétés non financières. Les sociétés dont l'activité principale est l'exploitation d'une affaire bancaire ou similaire, le financement du crédit aux entreprises ou aux consommateurs, le

1. Cette étude utilise le terme de sous-capitalisation dans le sens qui lui est généralement attribué. Un groupe d'entreprises est considéré comme sous-capitalisé lorsque son endettement est supérieur à celui des autres entreprises. Cette notion n'implique pas qu'il existe une norme de capitalisation optimale.

2. Nous remercions messieurs Gosselin et Taillon, du service des recherches statistiques du ministère du Revenu du Québec, qui ont permis la réalisation des traitements informatiques complémentaires requis par cette étude. 
courtage en valeurs mobilières, la gestion de placement, l'émission de polices d'assurance ou le commerce des valeurs mobilières sont exclues de l'étude, puisque leurs ratios d'endettement ne sont pas comparables à ceux des autres secteurs. Les statistiques sont calculées à partir d'un échantillon représentatif de 15853 sociétés (pour l'exercice 1990) ${ }^{3}$. La répartition de la population des sociétés en différents groupes de taille apparaît au tableau 1. Nous avons également retenu la distinction entre sociétés imposables et non imposables, utilisée par Revenu Québec. Cette distinction recoupe approximativement celle que l'on peut établir entre les sociétés qui dégagent des bénéfices et celles qui montrent des pertes. Le statut relativement à l'impôt est utilisé comme estimateur de la rentabilité, définie ici comme la capacité d'une entreprise à dégager un surplus ${ }^{4}$. Le tableau 1 montre qu'en 1990, 51,8\% des entreprises ne versaient pas d'impôt au Québec. Cette proportion est particulièrement élevée parmi les entreprises artisanales $(62 \%)$ et parmi les très grandes entreprises $(57,6 \%)$. L'échantillon est ainsi divisé en deux groupes sensiblement de même taille, du point de vue de la rentabilité fiscale (c'est-à-dire observée après application des règles fiscales et non comptables).

Les données sont agrégées, ce qui n'autorise pas les analyses détaillées et les tests statistiques. Les ratios sont calculés à partir des postes suivants : actif total, bénéfices non répartis, passif à court terme, dettes à long terme, dus aux actionnaires et autres passifs. Les prêts d'actionnaires sont assimilés aux fonds propres dans le calcul des ratios de capitalisation. En effet, souvent réalisés pour des motifs fiscaux ou pour améliorer la liquidité de l'investissement, ces prêts présentent toutes les caractéristiques des fonds propres: absence de garantie, échéancier de remboursement non défini, intérêt nul ou inférieur au taux du marché. De plus, leur remboursement est le plus souvent

3. Les données financières et fiscales présentées dans les Portraits de la Fiscalité des Corporations sont extraites des déclarations de revenu des entreprises et des états financiers qui y sont annexés. L'échantillonnage est réalisé selon un plan stratifié déterminé par deux critères : le secteur d'activité et le chiffre d'affaires. D'après Revenu Québec, «Les ressources allouées à la transcription permettent l'échantillonnage d'un nombre suffisant de corporations pour assurer la qualité des estimations » (Portrait de la Fiscalité des Corporations au Québec, 1989). Toutefois, de l'avis même des responsables de ces traitements, des problèmes d'estimation subsistent. Par exemple, pour l'année 1987, des incohérences dans les chiffres concernant les entreprises de plus de 200 millions de dollars d'actif ont conduit à exclure ces données de l'étude.

4. La correspondance n'est qu'approximative, en raison principalement des différences entre l'amortissement fiscal et l'amortissement comptable, qui permettent aux sociétés de réduire leur bénéfice imposable. Par ailleurs, il serait important de poursuivre cette étude en utilisant des mesures plus fines de la rentabilité des entreprises. Ces travaux, qui ne peuvent être entrepris à partir des données agrégées utilisées ici, sont en cours de réalisation. 
subordonné au remboursement des dettes externes. Ces prêts représentent le quart de l'actif dans le groupe des entreprises artisanales et 5 à $6 \%$ de celui des grandes entreprises ${ }^{5}$. Les autres passifs incluent le solde créditeur d'impôt reporté. Ce poste, qui devrait être intégré aux fonds propres (Gagnon et Papillon, 1984), n'a pu être isolé. Les fonds propres correspondent donc à la somme des bénéfices non répartis et du capital-actions souscrit et payé. La dette externe regroupe l'ensemble du passif à court terme et les dettes à long terme externes.

Les statistiques fiscales permettent de classer les entreprises suivant leurs ventes ou leur actif total. Ici, l'actif total a été préféré aux ventes comme critère de taille, puisque l'échantillon regroupe à la fois des entreprises manufacturières et commerciales, ce qui rend délicate l'estimation de la taille à l'aide des ventes. En dépit des difficultés que pose cette mesure (d'Amboise, 1993), la taille des entreprises est donc mesurée par l'actif.

Il faut finalement noter que les données proviennent de rapports préparés à des fins fiscales. Les niveaux d'endettement diffèrent donc de ceux que l'on pourrait estimer à partir de données comptables. Dans la mesure où le décalage entre l'endettement «comptable» et l'endettement «fiscal» affecte de la même manière les entreprises des différentes classes d'actif, les comparaisons présentées ici restent valables. Afin de déterminer dans quelle proportion l'emploi de ces données fausse l'évaluation de l'endettement, nous avons rapporté des estimations de l'endettement obtenues à l'aide d'un échantillon d'entreprises publiques. Après élimination des impôts reportés, les niveaux moyens d'endettement calculés sont proches de ceux estimés à partir des données fiscales.

\section{La capitalisation des entreprises: état de la situation}

\subsection{Taille et capitalisation}

La situation de l'endettement qui prévalait au Québec en 1990 est illustrée par les figures 1 à 3 . Les données détaillées apparaissent aux différents volets du tableau 1. Les entreprises ont été regroupées en dix classes suivant l'actif total.

La figure 1 montre qu'il n'existe pas de relation linéaire entre la taille et la capitalisation des entreprises, dès que les prêts d'actionnaires ou de filiales sont considérés comme des fonds propres, ce qu'ils sont effectivement.

5. Notre évaluation de la proportion du financement fournie sous forme de prêts par les actionnaires et les sociétés affiliées sous-évalue l'importance réelle de ce type de financement, puisque les prêts d'actionnaires à court terme n'ont pu être estimés. 
Seules les entreprises artisanales, dont l'actif est inférieur à 100000 dollars présentent un ratio des fonds propres à l'actif total inférieur à la moyenne de la distribution interclasse des ratios de capitalisation (0,392 par rapport à 0,428 ). Ce résultat semble lié à la très forte proportion de sociétés non imposables dans cette catégorie. Il apparaît en effet que les entreprises artisanales imposables sont fortement capitalisées $(0,538$ par rapport à 0,513 pour la moyenne des sociétés imposables).

Viennent ensuite trois catégories de petites entreprises fortement capitalisées qui rassemblent plus de 66000 observations. Ces entreprises, dont l'actif se situe entre 100000 et 1 million de dollars, sont, en moyenne, les moins endettées des entreprises québécoises. Les plus petites des PME québécoises ne sont donc pas sous-capitalisées ${ }^{6}$. Cela est particulièrement vrai pour les 28000 entreprises imposables de 100000 à 500000 dollars d'actif, dont le ratio des fonds propres à l'actif dépasse $56 \%$.

Inversement, on observe une capitalisation moins forte pour les quatre catégories suivantes, où l'endettement se situe en dessous de la moyenne générale. L'écart entre l'endettement moyen et celui de trois de ces catégories n'est cependant que de deux points de pourcentage. Seule la catégorie des entreprises de 5 à 15 millions de dollars d'actif semble souffrir d'un déficit de fonds propres. Ce phénomène est également observable lorsqu'on se limite aux sociétés imposables. Celles dont l'actif se situe entre 5 et 15 millions de dollars ont un ratio de capitalisation inférieur à celui des entreprises de petite taille (actif compris entre 100000 et 200000 dollars) par plus de 10 points de pourcentage. Il s'agit donc d'une caractéristique liée à cette catégorie d'actif et non à la fréquence plus élevée d'entreprises non rentables. Finalement, les grandes entreprises dont l'actif dépasse 200 millions de dollars présentent des taux de capitalisation supérieurs à la moyenne et à peu près égaux à ceux des entreprises de petite taille.

La figure 1 et le tableau 1 montrent donc que la relation entre la taille et l'endettement des entreprises québécoises n'est pas linéaire. Cette relation aurait la forme d'un $U$ très aplati, les entreprises les moins capitalisées se situant dans les classes d'actif de 1 à 50 millions. Cependant, il faut noter que les différences entre les niveaux moyens d'endettement des diverses classes sont, au maximum, de 7 points (entre $38,4 \%$ et $45,6 \%$ ). Comme le montrent

6. Le ministère du Revenu du Québec classe les entreprises de la façon suivante:

$\begin{array}{lll}\text { Taille } & \begin{array}{l}\text { Secteur manufacturier: } \\ \text { tranche d'actif }\end{array} & \begin{array}{l}\text { Autres secteurs: } \\ \text { tranche de revenus }\end{array} \\ \text { Petite } & \text { moins de } 3 \mathrm{M} \$ & \text { moins de } 2 \mathrm{M} \$ \\ \text { Moyenne } & \text { de } 3 \text { à } 12 \mathrm{MS} & \text { de } 2 \text { à } 20 \mathrm{M} \$ \\ \text { Grande } & 12 \mathrm{M} \$ \text { et plus } & 20 \mathrm{M} \$ \text { et plus }\end{array}$




\section{FIGURE 1}

Structures financières des entreprises non financières au Québec en fonction de leur actif total, en 1990

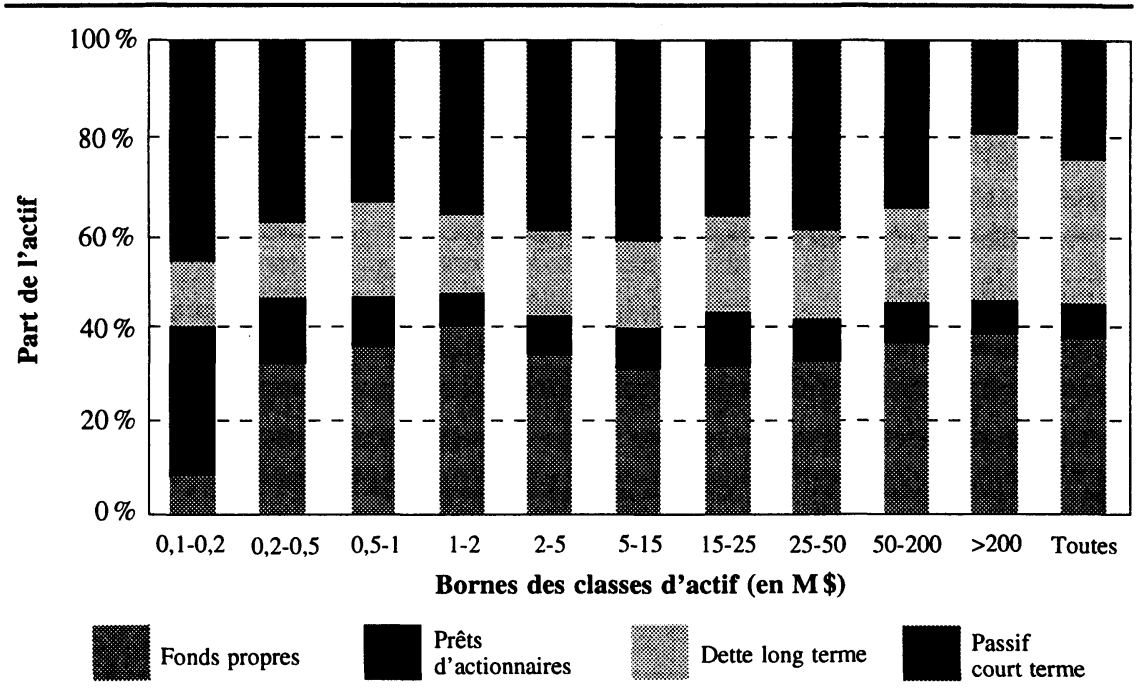

FIGURE 2

Structures financières des entreprises non financières imposables au Québec, en 1990

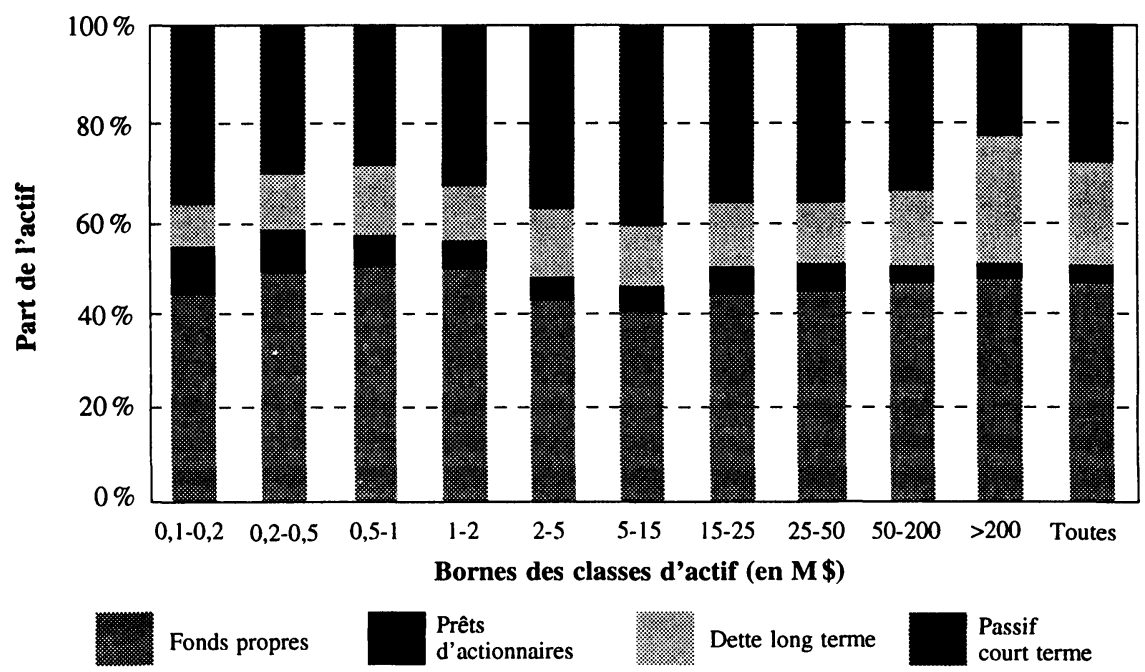


TABLEAU 1

Structures financières agrégées des entreprises non financières au Québec, en 1990, pour l'ensemble de la population puis en fonction de leur statut fiscal

\begin{tabular}{|c|c|c|c|c|c|c|c|c|c|c|c|c|}
\hline & \multicolumn{10}{|c|}{ Classes d'actif (en M \$) } & \multirow[t]{2}{*}{ Total } & \multirow[t]{2}{*}{$\begin{array}{c}\text { Sociétés } \\
\text { cotées }\end{array}$} \\
\hline & $<0,1$ & $0,1-0,2$ & $0,2-0,5$ & $0,5-1$ & $1-5$ & $5-15$ & $15-25$ & $25-50$ & $50-200$ & $>200$ & & \\
\hline \multicolumn{13}{|l|}{ Ensemble des sociétés } \\
\hline $\begin{array}{l}\text { Passif court terme }(\%) \\
\text { Dette long terme }(\%) \\
\text { Fonds propres }(\%) \\
\text { Nombre de firmes }(000) \\
\text { Actif total (milliards \$) }\end{array}$ & $\begin{array}{c}0,471 \\
0,137 \\
0,392 \\
72,54 \\
2,75\end{array}$ & $\begin{array}{r}0,380 \\
0,164 \\
0,456 \\
25,060 \\
3,540\end{array}$ & $\begin{array}{r}0,349 \\
0,195 \\
0,456 \\
26,220 \\
8,420\end{array}$ & $\begin{array}{r}0,372 \\
0,165 \\
0,463 \\
14,920 \\
10,460\end{array}$ & $\begin{array}{r}0,405 \\
0,186 \\
0,409 \\
14,790 \\
30,000\end{array}$ & $\begin{array}{r}0,427 \\
0,189 \\
0,384 \\
2,740 \\
23,530\end{array}$ & $\begin{array}{r}0,385 \\
0,193 \\
0,422 \\
0,730 \\
13,830\end{array}$ & $\begin{array}{r}0,404 \\
0,187 \\
0,409 \\
0,610 \\
21,770\end{array}$ & $\begin{array}{r}0,358 \\
0,201 \\
0,441 \\
0,590 \\
55,790\end{array}$ & $\begin{array}{r}0,203 \\
0,352 \\
0,445 \\
0,330 \\
373,410\end{array}$ & $\begin{array}{r}0,375 \\
0,197 \\
0,428 \\
158,530 \\
543,500\end{array}$ & $\begin{array}{r}0,220 \\
0,285 \\
0,505 \\
0,325 \\
1112,000\end{array}$ \\
\hline \multicolumn{13}{|l|}{ Sociétés imposables } \\
\hline $\begin{array}{l}\text { Passif court terme }(\%) \\
\text { Dette long terme }(\%) \\
\text { Fonds propres }(\%) \\
\text { Nombre de firmes }(000) \\
\text { Actif total (millions } \$ \text { ) }\end{array}$ & $\begin{array}{r}0,369 \\
0,093 \\
0,538 \\
27,320 \\
1,000\end{array}$ & $\begin{array}{r}0,309 \\
0,121 \\
0,571 \\
12,890 \\
1,840\end{array}$ & $\begin{array}{r}0,293 \\
0,143 \\
0,564 \\
15,320 \\
4,890\end{array}$ & $\begin{array}{l}0,335 \\
0,118 \\
0,547 \\
9,240 \\
6,580\end{array}$ & $\begin{array}{r}0,380 \\
0,147 \\
0,473 \\
9,140 \\
18,940\end{array}$ & $\begin{array}{r}0,416 \\
0,130 \\
0,454 \\
1,460 \\
12,320\end{array}$ & $\begin{array}{l}0,375 \\
0,133 \\
0,492 \\
0,360 \\
6,870\end{array}$ & $\begin{array}{l}0,374 \\
0,127 \\
0,499 \\
0,270 \\
9,870\end{array}$ & $\begin{array}{r}0,346 \\
0,159 \\
0,495 \\
0,290 \\
21,270\end{array}$ & $\begin{array}{r}0,234 \\
0,270 \\
0,496 \\
0,140 \\
125,550\end{array}$ & $\begin{array}{r}0,343 \\
0,144 \\
0,513 \\
76,430 \\
209,130\end{array}$ & \\
\hline \multicolumn{13}{|l|}{ Sociétés non imposables } \\
\hline $\begin{array}{l}\text { Passif court terme }(\%) \\
\text { Dette long terme }(\%) \\
\text { Fonds propres }(\%) \\
\text { Nombre de firmes }(000) \\
\text { Actif total (millions } \$ \text { ) }\end{array}$ & $\begin{array}{r}0,553 \\
0,172 \\
0,275 \\
45,220 \\
1,530\end{array}$ & $\begin{array}{r}0,457 \\
0,211 \\
0,332 \\
12,180 \\
1,698\end{array}$ & $\begin{array}{r}0,425 \\
0,267 \\
0,308 \\
10,890 \\
3,530\end{array}$ & $\begin{array}{l}0,434 \\
0,244 \\
0,322 \\
5,680 \\
3,880\end{array}$ & $\begin{array}{r}0,446 \\
0,248 \\
0,306 \\
5,660 \\
12,010\end{array}$ & $\begin{array}{r}0,439 \\
0,254 \\
0,307 \\
1,290 \\
11,210\end{array}$ & $\begin{array}{l}0,395 \\
0,251 \\
0,354 \\
0,370 \\
6,960\end{array}$ & $\begin{array}{r}0,430 \\
0,237 \\
0,333 \\
0,330 \\
11,890\end{array}$ & $\begin{array}{r}0,369 \\
0,242 \\
0,389 \\
0,300 \\
28,520\end{array}$ & $\begin{array}{r}0,188 \\
0,394 \\
0,419 \\
0,190 \\
247,870\end{array}$ & $\begin{array}{r}0,414 \\
0,252 \\
0,335 \\
82,110 \\
329,098\end{array}$ & \\
\hline
\end{tabular}


FIGURE 3

Structures financières des entreprises non financières non imposables au Québec, en 1990

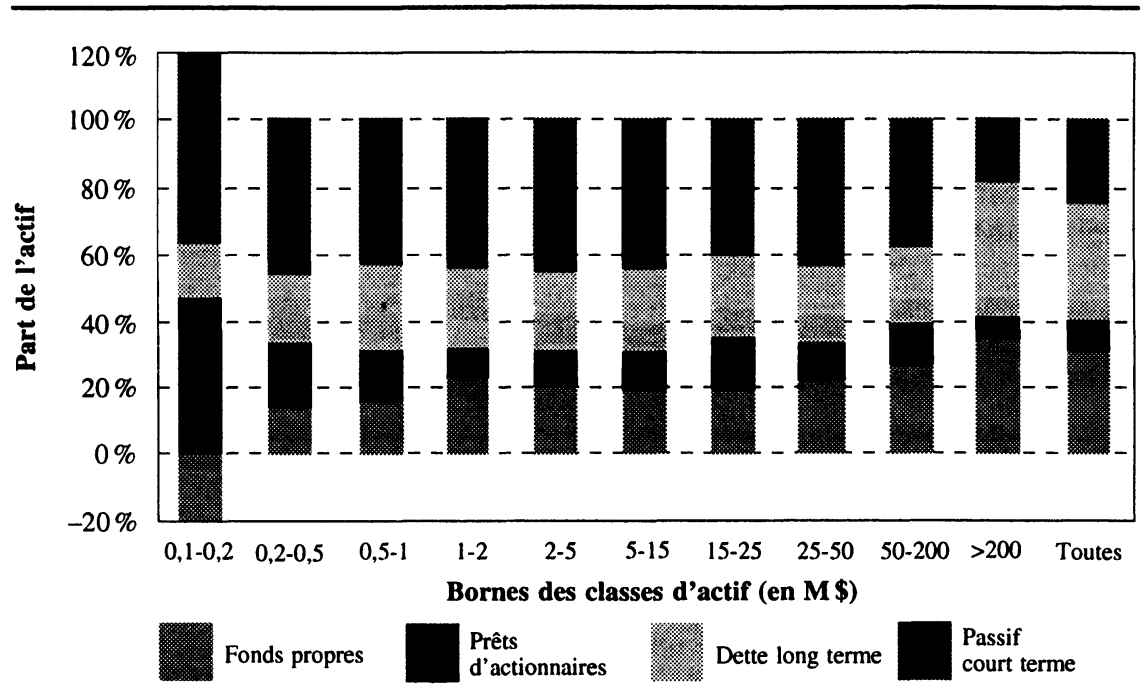

les figures 2 et 3, la rentabilité induit des différences d'endettement nettement plus grandes que ne le fait la taille des entreprises. L'observation selon laquelle les entreprises de petite taille sont mieux capitalisées que les plus grandes est parfaitement cohérente avec les résultats de Caldwell, Sawchuk et Wilson (1994). À partir de la base de microdonnées de Statistique Canada, qui comporte plus de 25000 observations, ils notent (p. 27) que ce sont les entreprises les plus petites qui utilisent le plus le capital-actions.

\subsection{La relation rentabilité-endettement}

Les figures 2 et 3 présentent la relation entre les structures financières et la taille en fonction de l'imposition des sociétés. Les sociétés imposables, représentées à la figure 2 , sont faiblement endettées. Leur ratio moyen de capitalisation est de l'ordre de $51,3 \%$, et il atteint $57 \%$ pour certaines catégories de petites entreprises. Cette constatation tient pour toutes les classes, y compris les entreprises artisanales. Ici encore, les petites entreprises apparaissent sensiblement moins endettées que les entreprises moyennes et la relation prend une forme en U. La situation s'avère fort différente pour les entreprises non imposables qui sont beaucoup plus endettées. Leur capitalisation moyenne se situe autour de $33,5 \%$. L'écart entre l'endettement des entreprises suivant 
qu'elles sont ou non imposables atteint 25 points de pourcentage dans certaines classes et semble diminuer avec la taille. L'effet de la rentabilité sur l'endettement serait donc d'autant plus fort que l'entreprise est petite. Cette observation peut s'expliquer par un recours plus facile au financement par fonds propres externes pour les grandes entreprises. Dans le cas des PME, où le réinvestissement des bénéfices constitue souvent la seule source possible d'augmentation des fonds propres, il s'établit une relation directe entre les bénéfices et l'endettement.

Les entreprises artisanales présentent un déficit accumulé qui atteint près de $20 \%$ de l'actif total. L'endettement excède donc $100 \%$. Comme l'ont déjà montré Whipp et al. (1982), l'inclusion des entreprises artisanales dans la catégorie des PME influence fortement les résultats des analyses comparatives des structures financières. À l'inverse, leur exclusion permet de remettre en cause l'affirmation selon laquelle les petites entreprises sont globalement souscapitalisées. Quelle que soit la taille des entreprises, la sous-capitalisation est donc avant tout le résultat d'une faible rentabilité.

\section{L'évolution de l'endettement}

Compte tenu des similitudes observées dans les caractéristiques de certaines catégories d'entreprises, nous avons composé quatre groupes pour analyser l'évolution de l'endettement, qui est illustrée par la figure 4. Le premier ensemble regroupe les entreprises artisanales, dont l'actif ne dépasse pas 100000 dollars. Le second inclut les entreprises de 100000 dollars à 1 million de dollars d'actif. Elles sont qualifiées de petites entreprises. La troisième classe est composée des entreprises dont l'actif se situe entre 1 et 15 millions de dollars d'actif et qui, d'après des analyses effectuées en 1990, apparaissaient comme les plus endettées. Les grandes entreprises sont celles dont l'actif dépasse 15 millions et une cinquième catégorie d'entreprises a été ajoutée aux fins de validation. Il s'agit des entreprises inscrites à la Bourse de Toronto et suivies par Compustat. Cet échantillon comporte, suivant les années, de 250 à 350 titres, qui constituent le sous-ensemble des titres les plus capitalisés transigés sur le parquet de la Bourse de Toronto (le TSE) ${ }^{7}$.

En premier lieu, on observe peu de différences entre l'évolution des grandes entreprises québécoises et celle des titres du TSE, sauf pour les deux

7. Afin de rendre comparables les ratios d'endettement calculés à partir des données comptables de Compustat et ceux tirés des rapports d'impôt, le solde créditeur d'impôt reporté a été enlevé du bilan des entreprises du TSE. Cette correction élimine partiellement les distorsions introduites par les différences entre l'amortissement fiscal et comptable dans le bilan des sociétés. 


\section{FIGURE 4}

Évolution de l'endettement total des entreprises québécoises, comparé à celui des grandes entreprises inscrites à la Bourse de Toronto, 1979-1990

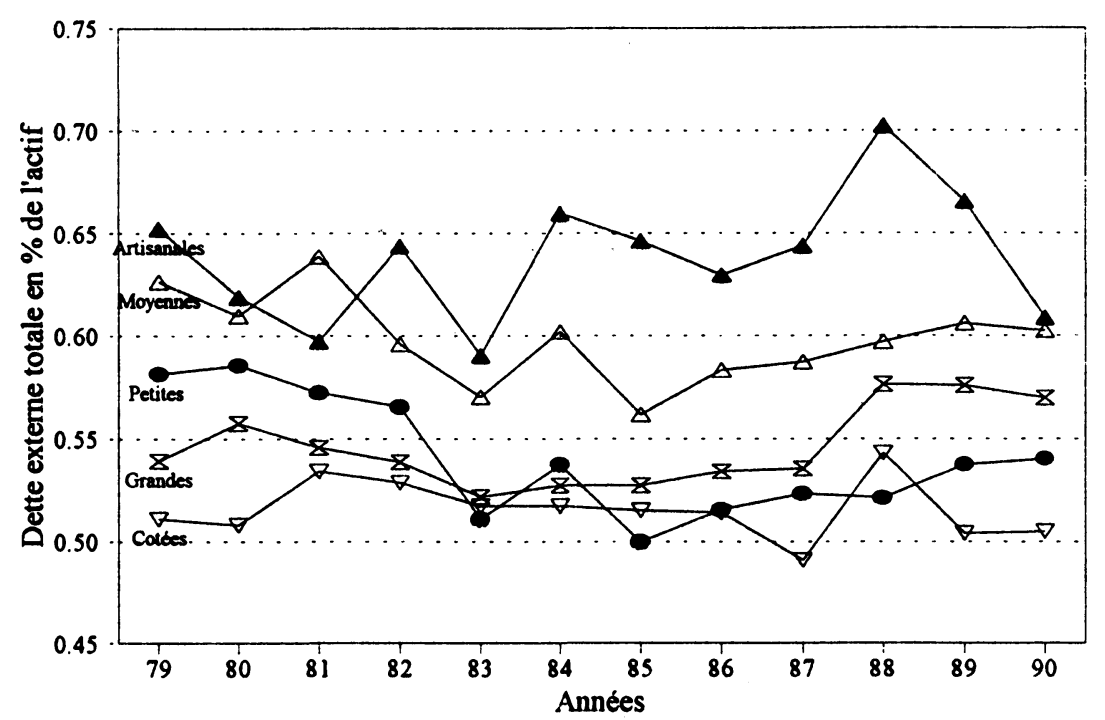

Légende :

Sociétés artisanales: moins de $100000 \$$ d'actif Petites sociétés : de 100000 à 1000000 \$ d'actif Sociétés moyennes: de 1000000 à $15000000 \$$ d'actif Grandes sociétés: de 15000000 à $200000000 \$$ d'actif Sociétés cotées : échantillon de Compustat

dernières années de la période d'analyse. L'emploi de données fiscales ne semble donc pas invalider notre analyse de l'endettement. En second lieu, les petites entreprises (dont l'actif se situe entre 100000 dollars et 1 million de dollars) réduisent leur endettement depuis le début de la période d'observation. Elles étaient, en 1979, moins endettées que les entreprises de taille moyenne et les entreprises artisanales, et sont, depuis 1984, les moins endettées de toutes les catégories d'entreprises. L'argument de la sous-capitalisation ne tient donc pas pour les petites entreprises en 1990, pas plus d'ailleurs qu'il ne tenait en 1979.

Pour les entreprises de taille moyenne, la tendance générale semble être à la réduction de l'endettement total: le ratio d'endettement est passé de 63 à $60 \%$ environ, entre 1979 et 1990 . Cependant, on note une augmentation depuis 1985, qui est également observable dans le groupe des grandes entreprises. 


\section{FIGURE 5}

\section{Évolution du ratio d'endettement à long terme des entreprises québécoises, 1979-1990}

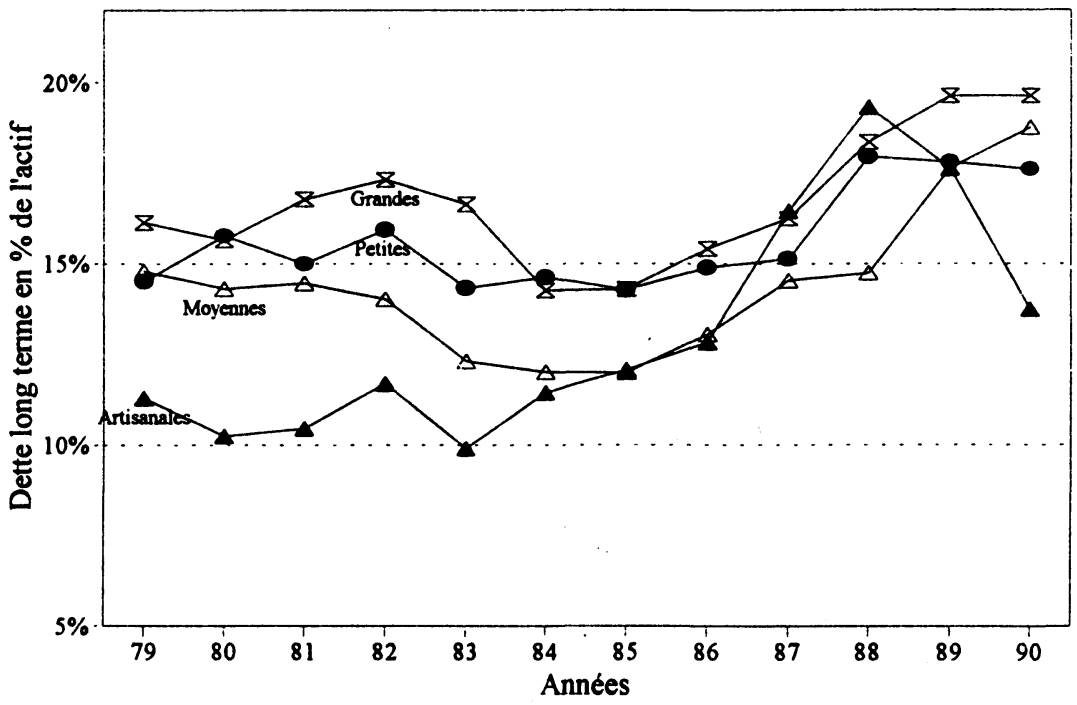

Légende :

Sociétés artisanales: moins de $100000 \$$ d'actif

Petites sociétés: de 100000 à $1000000 \$$ d'actif

Sociétés moyennes: de 1000000 à $15000000 \$$ d'actif

Grandes sociétés: de 15000000 à $200000000 \$$ d'actif

Finalement, les entreprises artisanales semblent avoir un comportement erratique dont il est difficile de dégager une tendance significative.

La relative stabilité des niveaux d'endettement occulte, cependant, des effets de substitution entre les types d'endettement qui sont illustrés par les figures suivantes. La figure 5 montre, pour toutes les catégories d'entreprises à l'exception des plus grandes, une réduction importante du passif à court terme en proportion de l'actif total. Pour les entreprises de taille moyenne, cette proportion est passée de 48 à $42 \%$, alors que les valeurs correspondantes sont de 44 et $36 \%$ dans le cas des entreprises de petite taille. La figure 6 montre que cette réduction du passif à court terme coïncide avec une augmentation de la part du financement représentée par la dette à long terme. La structure de l'endettement des sociétés québécoises a donc évolué au cours de la décennie, mais le niveau global d'endettement n'a que légèrement diminué pour l'ensemble des entreprises, à l'exception des plus grandes qui se tournent davantage vers l'endettement. 


\section{FIGURE 6}

Évolution du ratio du passif à court terme des entreprises québécoises, 1979-1990

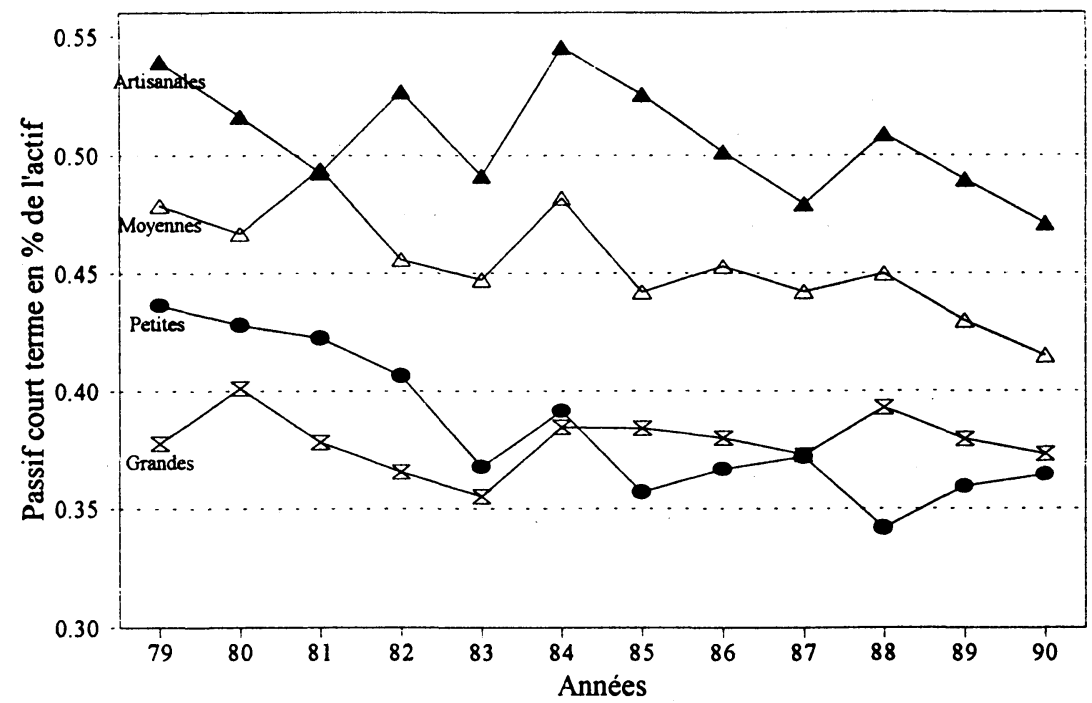

Légende :

Sociétés artisanales: moins de $100000 \$$ d'actif

Petites sociétés: de 100000 à $1000000 \$$ d'actif

Sociétés moyennes: de 1000000 à $15000000 \$$ d'actif

Grandes sociétés : de 15000000 à $200000000 \$$ d'actif

\section{Conclusion}

Cette analyse de la situation et de l'évolution de l'endettement des PME conduit à distinguer plusieurs groupes d'entreprises au sein même de l'ensemble des PME. Les entreprises artisanales sont fortement endettées. Ce résultat est en grande partie imputable à la présence d'un fort contingent de sociétés non imposées, et donc vraisemblablement non rentables. Celles-ci présentent un endettement supérieur à $100 \%$. Les petites entreprises dont l'actif se situe entre 100000 dollars et 1 million de dollars d'actif ont, en 1990, un niveau d'endettement inférieur à celui de toute autre catégorie d'entreprises et ne présentent aucun symptôme de sous-capitalisation. Les entreprises de taille moyenne sont davantage endettées, et cette constatation est particulièrement vraie pour les sociétés non imposables. Il faut, par ailleurs, noter que l'écart maximal observé entre les niveaux d'endettement de catégories d'entreprises formées selon la taille est de l'ordre de 7 points alors que des écarts de 15 à 25 points séparent les sociétés de même taille suivant qu'elles sont ou non imposables. La 
rentabilité a donc une bien meilleure valeur prédictive de l'endettement que la taille.

Les résultats de l'étude confirment les observations faites à l'échelle canadienne: les PME sont mieux capitalisées que les entreprises de grande taille. Ils indiquent plusieurs voies de recherche que pourront explorer les études qui utilisent des données désagrégées. En premier lieu, la situation canadienne semble différer de celle qui semble prévaloir dans d'autres pays, notamment en France où plusieurs travaux font état d'un endettement plus lourd des entreprises de petite taille (Levratto, 1990; Belletante, 1991). Il serait important de déterminer dans quelle mesure ces divergences découlent de différences entre les pratiques de gestion, les caractéristiques de l'environnement ou, tout simplement, entre les devis de recherche. En second lieu, la relation entre la taille et l'endettement est complexe et non linéaire, puisque l'effet de la rentabilité sur l'endettement semble être une fonction de la taille. Des travaux plus approfondis sont donc requis afin de mieux comprendre l'origine et la nature de ces relations. Il sera également important de déterminer l'origine de l'endettement plus élevé observé dans le groupe des entreprises de taille moyenne.

Pour les politiques économiques, les résultats de ce travail sont également clairs. La petite entreprise n'est pas sous-capitalisée et la solution des problèmes de financement des PME, s'ils existent, ne passe vraisemblablement pas par l'augmentation démesurée de l'offre de capital de risque subventionné. L'expérience québécoise montre, en effet, clairement que la majeure partie de ce capital ne peut être investie. Il existe toutefois dans cette catégorie des entreprises peu rentables dont l'endettement augmente rapidement, puisqu'elles ne génèrent pas les bénéfices dont le réinvestissement constitue la principale source d'augmentation des fonds propres. Le problème de ces entreprises se situe donc au niveau de la rentabilité et non au niveau de l'accès aux sources de financement. Le déficit de fonds propres apparaît principalement pour les entreprises dont l'actif se situe entre 5 et 15 millions de dollars. Ce résultat est surprenant, puisqu'il s'agit d'une catégorie d'entreprises pour lesquelles il existe une offre de capital de risque considérable. Il faut donc étudier pourquoi il semble exister simultanément sur le marché financier du Québec une demande apparente de fonds propres et un excédent d'offre. Cette question fera l'objet de travaux ultérieurs. 


\section{Bibliographie}

Belletante, B. (1991), «Pour une approche des spécificités financières de la PME au travers du concept de territoire financier», Revue Internationale PME, vol. $4, \mathrm{n}^{\circ} 1$.

Caldwell, D., G. SawchuK et J. Wilson (1994), «Do small firms face higher financing costs?», Statistique Canada, $\mathrm{n}^{\circ}$ 61-008.

CONSEIL DE LA SCIENCE ET DE LA TECHNOLOGIE (1993), Urgence technologique: pour un Québec audacieux, compétitif et prospère, Québec.

GAGNON, J.M. et B. PAPILlON (1984), Risque financier, taux de rendement des entreprises canadiennes et intervention de l'État, Conseil économique du Canada, Ottawa.

D'Amвoise, G. (1993), La PME canadienne: situation et défis, Institut de recherches politiques, Sainte-Foy, Québec, Les Presses de l'Université Laval.

LeVratto, N. (1990), «Le financement des PME par les banques: contraintes des firmes et limites de la coopération", Revue Internationale PME, vol. $3, \mathrm{n}^{\circ} 2$, p. 193-213.

Ministère de L'INdustrie, du CoMmerCe et DE LA TeChNologie (1988), Les PME au Québec: état de la situation, Gouvernement du Québec, Québec.

REVENU QUÉBEC, Statistiques financières et fiscales des sociétés, Gouvernement du Québec, Québec, diverses années.

WHIPP, J.E., HUGUES et J.R. D'CRUZ (1982), «A profile of small business in Canada: a background report», for the Small Business Review Team, Department of Industry, Trade and Commerce, Ottawa, mai, 77 pages. 